DOI: $10.25100 /$ pfilosofica.v0i49.8053

\title{
LA SOLEMNIDAD DE UN ROSTRO DE ARENA
}

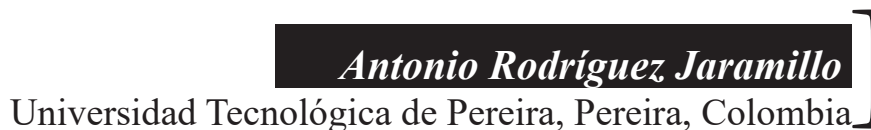

\section{Resumen}

Confusa, difusa, profusa, la «muerte del hombre» después de cinco décadas de su proclamación sigue siendo una cuestión punzante para no pocos lectores de Foucault. Sin embargo, llama la atención que la mayoría de esos preocupados lectores no considera las rectificaciones que el autor hace a esa formulación. Examinar esas rectificaciones, en relación con los desplazamientos del pensamiento de Foucault, es el propósito de estas reflexiones e inflexiones.

Palabras clave: muerte del hombre; antropología; subjetividad; técnicas de sí; genealogía.

Cómo citar este artículo: Rodríguez-Jaramillo, A. (2019). La solemnidad de un rostro de arena. Praxis Filosófica, (49), 171 - 190. doi: 10.25100/pfilosofica.v0i49.8053

Recibido: 19 de enero de 2019. Aprobado: 03 de marzo de 2019. 


\title{
Solemnity of a Sand-Made Face
}

\begin{abstract}
Antonio Rodríguez Jaramillo ${ }^{I}$
Abstract

Confused, diffuse, profuse, the "death of man» remains a stabbing issue for many Foucault readers after five decades of its proclamation. However, it is striking that most of these concerned readers do not consider the author's rectifications to that formulation. To examine these rectifications, in relation to the displacements of Foucault's thought, is the purpose of these reflections and inflections.
\end{abstract}

Keywords: Man's death; Anthropology; Subjectivity; Technologies of the Self; Genealogy.

${ }^{1}$ Universidad Tecnológica de Pereira, profesor titular. Doctor en filosofía del Instituto de Filosofía de la Universidad de Antioquia; licenciado y magister en filosofía de la Universidad del Valle. Ha publicado los libros Montaigne. Ética en diálogos modernos: los Ensayos; "Yo soy un moralista". (2017). Foucault, las alethurgias. Praxis Filosófica, (45), 145-169. Áreas de trabajo: historia de la filosofía, escepticismo, ética.

ORCID: 0000-0001-7615-7352 E-mail: antor@utp.edu.co 


\title{
LA SOLEMNIDAD DE UN ROSTRO DE ARENA
}

\author{
Antonio Rodríguez Jaramillo \\ Universidad Tecnológica de Pereira, Pereira, Colombia
}

\section{Introducción}

Con solemnidad, dos años antes de Mayo del 68, Foucault dice: "[...] se puede apostar que el hombre se borrará, como en el borde del mar un rostro de arena" (Foucault, 1966, p. 398). Sin dramatismo, doce años después, manifiesta: "En Las palabras y las cosas, yo me he equivocado presentando esta muerte como algo que estaba en curso en nuestra época. Yo he confundido dos aspectos [...] más yo no cedo en el fondo" (Foucault, 2001a, p. 894). ¿Bajo qué condiciones Foucault reconoce haberse equivocado presentado la muerte del hombre como un acontecimiento que estaba acaeciendo en nuestra actualidad y, a la vez, afirma no ceder en el fondo? Consideramos que esas condiciones se envuelven en una nueva manera de asumir la historia del presente. En los últimos años de la década del 70, Foucault descubre un modo otro de enfrentar la historia de la actualidad. Se trata de una "modestia del presente" que desplaza la "solemnidad" con que se enunciaba un acontecimiento tomado como constitutivo de una actualidad que rompe radicalmente con el pasado. Este nuevo modo de afrontar las experiencias históricas conducen, por una parte, a transformar la muerte del hombre en una metáfora que solamente designa un rechazo -por ejemplo, a una naturaleza humana, a una identidad que puede ser alienada,-; y, por otra, a reinscribir "el hombre" como un episodio en la larga historia de las técnicas de sí inventadas por el cristianismo de los primeros siglos. La sustentación de este punto de vista se envuelve en las páginas siguientes. 


\section{La solemnidad del presente}

Confusa, difusa, profusa, la muerte del hombre ha sido objeto de diversas interpretaciones; varios estatutos y roles se le ha asignado en el pensamiento de Foucault; tampoco ha faltado quienes redujeron las complejas problemáticas de Las palabras y las cosas a esa muerte ni quienes hicieron de ella una especie de slogan del pensamiento de su autor. Sin embargo, que la mayoría de esos preocupados lectores no consideren las rectificaciones que Foucault hace a esa proclamación es notorio ${ }^{2}$, como también que se tenga poco en cuenta el significativo cambio de perspectiva sobre la historia del presente, de la actualidad, que efectúa el filósofo en la segunda mitad de la década del $70^{3}$. El rugido de la crítica del sujeto fundador en el despertar del sueño antropológico es el título de un artículo (Nigro, 2012) que, enfocado en la muerte del hombre, parte del presupuesto: "[...] la deconstrucción del sujeto como arché [...] y de la reconstrucción de la subjetividad como efecto" (p. 60). Ese título parece convocar dos nombres: el primer segmento podría considerarse un eco de la figura nietzscheana del león, en las tres transformaciones del espíritu (Así hablaba Zarathustra). Rugiendo para conquistar su libertad, el león («un acto feroz que ha hecho una bestia de presa») inauguraría el pensamiento de Foucault. Un corolario se enuncia: "Cuestión de la que, sin duda, Foucault jamás se ha desembarazado en todo su recorrido filosófico" (Nigro, 2012, p. 70). El segundo segmento parece un eco de la figura kantiana del despertar del sueño de la metafísica; más si, para Heidegger, la antropología es una metafísica de la subjetividad, se infiere que el despertar efectivo sería del sueño antropológico. En ese despertar rugiría la crítica de Foucault, conducido por Nietzsche y Heidegger, y de los cuales no habría podido jamás soltarse. Nigro muestra claramente que el despertar del sueño antropológico se da en

\footnotetext{
${ }^{2}$ Por ejemplo, R. Castro se esfuerza por salvar la polémica formulación que cierra Las palabras y las cosas: "Foucault utiliza la expresión "quizá" para referirse al hipotético fin próximo del hombre [...] pero cuya realización completa se encuentra pendiente y puesta entre paréntesis" (Castro, 2005). Sin embargo, decir que "la realización completa..." implica de alguna manera que se está realizando. Por su parte, E. Castro, visita con frecuencia a la muerte del hombre situándola bajo el foco del lenguaje o los saberes, que luego Foucault desplazaría: "Foucault no proclama la muerte de la historia, pero, al menos la historia del conocimiento, en la medida en el que el sujeto ha muerto, debe convertirse en arqueología" (Castro, 2006).

${ }^{3}$ Ese cambio de perspectiva afecta esa mirada de la historia que se permite la formulación de expresiones tales como las que se leen en Las palabras y las cosas: “AAcaso es necesario presentir allí el nacimiento o, al menos aún, el primer fulgor bajo el cielo de un día que apenas se anuncia, pero donde ya adivinamos que el pensamiento - este pensamiento que habla desde hace miles de años sin saber lo que es hablar y ni siquiera que él habla- va a recogerse por entero y a iluminarse de nuevo en la claridad del ser?” (Foucault, 1966, p. 317).
} 
Francia -años 60- en una coyuntura marcada por el entrecruzamiento de la crítica y la antropología ( $c f$. Nigro, 2012, p. 62). El análisis se soporta sobre una operación: "[...] ahora yo quisiera privilegiar ciertos aspectos que se refieren más específicamente a la reflexión foucauldiana y al lugar que, en su trayectoria, le acuerda a la filosofía de Nietzsche" (Nigro, 2012, p. 62). «Privilegiar ciertos aspectos...»: no considerar otros. Nietzsche y Heidegger son dos pensadores «esenciales» para Foucault: su acceso a Nietzsche está mediado por su lectura de Heidegger, y su entrecruzamiento le abre el camino que conduce a la crítica histórica del sujeto soberano; de Heidegger procede su preocupación por el lenguaje, la antropología, las technai, entre otros puntos; a Nietzsche reconoce el mérito de sustituir en los análisis el sujeto fundador por el recurso a la historia. Pero, su relación con ellos, como «instrumentos del pensamiento», no permaneció siempre igual en su marcha filosófica. Enfoquemos un punto mayor: la posición histórico-filosófica que asume solemne y dramáticamente el presente, Foucault la recibe de Nietzsche ${ }^{4}$ y de Heidegger. Ese heroísmo del presente, sostenido y puesto en juego por el autor de Las palabras y las cosas en los análisis efectuados en los años 60, será sometido a un desplazamiento importante. Bajo esta perspectiva examinemos la muerte del hombre. La muerte del hombre es anunciada en relación con la muy reciente invención del hombre como sujeto y objeto de un saber posible. En el saber clásico, la figura del hombre no tenía presencia, ahí existían las representaciones ordenadas en el interior de un discurso, se encontraba el poder propio del discurso de representar el orden de las cosas. Por ejemplo, el estudio de la gramática o del orden de las riquezas pasaba por el discurso, y no por una ciencia del hombre. El ser del lenguaje se plegaba en la representación. Entre fines del siglo XVIII y principios del siglo XIX, el discurso pierde su papel de organizador de las representaciones, desaparece la transparencia entre el orden de las cosas y las representaciones; las cosas se repliegan en su propio espesor. Aparecen nuevas formas del saber, los lenguajes con su historia, la vida con su propia organización y autonomía, el trabajo con su propia capacidad de producción. Las cosas se amarran a su identidad con un lazo escondido por su historicidad. Por ejemplo, detrás de un ser que vive se arrastran largas

\footnotetext{
${ }^{4}$ Lo que dice J. P. Margot respecto a la genealogía vale también para el trabajo arqueológico: "El discurso histórico-político de la Genealogía pretende capturar el aspecto singular y enteramente diferente del momento presente. Hace, además, de la actividad filosófica de diagnosticar el presente un análisis concebido en términos de afinamiento de la conciencia de la discontinuidad donde el presente, o la modernidad, aparece como un determinado período de la historia, distinto de los demás períodos en virtud de unas características inherentes, o separado de los demás por algún acontecimiento dramático" (Margot, 2015, p. 201).
} 
cadenas irregulares de la vida que enrollan procesos orgánicos; detrás de las palabras hay estratos caóticos de significaciones sedimentadas. El lenguaje deja un espacio vacío del que emerge la figura del hombre, constituido como el aquel que vive, aquel que habla, aquel que trabaja; él es también aquel que conoce la vida, el lenguaje, el trabajo, y también el que puede ser conocido en cuanto que vive, habla, trabaja. En el vacío que deja el discurso de las representaciones, el hombre emerge en una analítica de la finitud que piensa lo finito a partir de lo finito. El hombre aparece y es manifestado por la positividad del saber en cuanto objeto finito de un saber posible y sujeto finito de conocimiento, disposición antropológica de los saberes. "Lo que Foucault designa como nacimiento del hombre es, por lo tanto, ese momento donde el saber moderno se ordena bajo la figura del hombre" (Gros, 2007, p. 42). La reconstrucción arqueológica del hombre pone ante los ojos su reciente invención histórica (efectivamente, Foucault no deconstruye el sujeto, como algunos comentaristas lo sostienen al ponerle la categoría derridiana). Con base en la reconstrucción de la reciente historia del hombre, apoyado en su diagnóstico del presente y entusiasmado con discursos nuevos (como lo ha mostrado De Libera en sus dos últimos cursos en el Collège de France, sobre Heidegger y Foucault) y las nuevas olas de la literatura, Foucault rebasa significativamente los límites de las investigaciones arqueológicas: "el hombre es una invención cuya fecha reciente muestra con toda facilidad la arqueología de nuestro pensamiento. Y quizá también su próximo fin [...] entonces podría apostarse a que el hombre se borrará, como en los límites del mar un rostro de arena". La figura del hombre desaparecerá en cuanto que la disposición del saber y el ser del lenguaje están en transformación a partir de ciertos acontecimientos discursivos que emergen desde el siglo XIX. Sin embargo, esa formulación, como también ese cierto trascendentalismo suprahistórico que Foucault le asigna al ser desnudo del lenguaje, será sometida a una recia rectificación genealógica. En 1978, Foucault dice:

Cuando hablo de muerte del hombre, quiero poner término a todo lo que quiere fijar una regla de producción, una meta esencial a esta producción del hombre por el hombre. En Las palabras y las cosas, me he equivocado al presentar esta muerte como algo que estaba en curso en nuestra época. He confundido dos aspectos. El primero es un fenómeno de pequeña escala [...]. Si la promesa de las ciencias humanas había sido hacernos descubrir el hombre, ellas no lo habían ciertamente alcanzado; pero, como experiencia cultural general, se trataba mejor de la constitución de una nueva subjetividad a través de la operación de reducción del sujeto humano a un objeto de conocimiento. 
El segundo aspecto, que he confundido con el precedente, es que en el curso de su historia los hombres no han cesado jamás de construirse ellos mismos, es decir, de desplazar continuamente su subjetividad, de constituirse en una serie infinita y múltiple de subjetividades diferentes y que jamás tendrán fin y que no nos pondrán jamás frente a algo que sería el hombre [...]. Hablando de la muerte del hombre, de modo confuso, simplificador, esto era lo que quería decir; más yo no cedo en el fondo. Es ahí donde hay incompatibilidad con la escuela de Frankfort. (Foucault, 2001a, p. 894).

Primero, muerte del hombre designa una voluntad de poner fin a todo aquello que pretende fijar una regla o una meta esencial a la producción del hombre por el hombre: terminar con aquello que, bajo una esencia del hombre, fija a éste como meta y prescribe el camino para alcanzarlo. A fines de 1978 (cuando la genealogía y la arqueología de la subjetividad contemporánea sitúa sus orígenes en la antigüedad griega y el cristianismo de los primeros siglos), muerte del hombre ha devenido en una metáfora. Segundo, la muerte del hombre como acontecimiento no está en curso en nuestra época; por consiguiente, se hablaría de un acontecimiento histórico que no está aconteciendo, o no acontece. Tercero, la equivocación proviene de la confusión de dos aspectos diferentes: uno, las ciencias humanas habían prometido hacer conocer al hombre esencial a través de los diferentes estudios que se daban al hombre como objeto de un saber posible, pero jamás lo lograron ni se aproximaron. La incumplida promesa, su fracaso eminente, es una de las razones que conlleva a Foucault a poner en cuestión el estatuto del hombre esencial, sus valoraciones, las reglas que impone y sus posibilidades futuras. Pero, de lo que se trataba era de plantear la cuestión del hombre como una experiencia cultural de la constitución de una nueva subjetividad a través de la operación de reducción del sujeto humano a un objeto de conocimiento. A ese fondo, sin simplificaciones y sin confusiones, solamente accede Foucault después de la segunda mitad de la década del 70. El otro aspecto, confundido con el anterior, radica en que en el curso de la historia, los hombres jamás nos han situado ni nos situarán frente a algo que sería el hombre esencial; puesto que, a lo largo de la historia, no han cesado de construirse ellos mismos, de desplazar continuamente su subjetividad y de constituirse en una serie infinita y múltiple de subjetividades diferentes que no tendrán jamás fin. Perpetuamente los hombres se empeñan en un proceso que, constituyendo objetos, los desplaza al mismo tiempo, los transforma y lo transfigura en sujeto. Cuarto, con base en lo anterior, se entiende efectivamente lo que Foucault quería decir (según sus palabras) con muerte del hombre; más se entiende también que a tal precisión y claridad, 
solamente accede después de un poco más de una década de la escritura de La palabras y las cosas. La equivocación, la confusión y las rectificaciones a la muerte del hombre son recias: muerte del hombre deviene en otra cosa, en una metáfora que designa el rechazo a una naturaleza del hombre, o a una identidad que puede ser objeto de alienación, represión o explotación o liberación, a la idea de una producción del hombre por el hombre a la manera la producción del valor, la riqueza, o de un objeto de uso económico (al modo de las tendencias marxistas de su época), con lo cual, Foucault se opone a la escuela de Frankfort. Tales puntos proceden de una cuestión que juega un papel relevante en los desplazamientos del pensamiento de Foucault. Nietzsche, o la epifanía:

[...] Nietzsche ha encontrado el punto donde el hombre y Dios se pertenecen el uno al otro, donde la muerte del segundo es sinónimo de la desaparición del primero [...] Nietzsche, proponiéndonos ese futuro a la vez como chance y como tarea, marca el umbral a partir del cual la filosofía contemporánea puede recomenzar a pensar [...]. Si bien el descubrimiento del Retorno es el fin de la filosofía, el fin del hombre es el retorno del comienzo de la filosofía [...] no es nada más, nada menos, que el despliegue de un espacio donde es por fin de nuevo posible pensar. La antropología [...] está en camino de disociarse bajo nuestros ojos, pues, nosotros comenzamos a reconocerla, a denunciarla bajo un modo de crítica, a la vez olvido de la abertura que la ha vuelto posible, y obstáculo terco que se opone obstinadamente a un pensamiento próximo (Foucault, 1966, p. 353).

La muerte del hombre es la proclamación solemne del fin histórico del sujeto trascendental que, bajo la disposición de la antropología, ha conducido el pensamiento hasta nuestra época; fin de un a priori que sirve de fundamento y de un primer comienzo a toda experiencia. Por esos tiempos, Una arqueología de las ciencias humanas concluye en el fin de una noche oscura y abre el comienzo de una nueva aurora del pensamiento. Una diferenciación se hace necesaria: hacer una arqueología de las ciencias humanas no implica en absoluto que el pensamiento del sujeto fundador sea la noche oscura de la cultura, ni que su desaparición sea el advenimiento de un espacio donde por fin es posible volver a pensar, ni que su desaparición se ha de producir. Allá subyace algo que no va por sí; en los bajos fondos del pensamiento, una imagen impensada juega su papel. ¿Qué idea es esa? En la década del 60, bastante afectado por las miradas de Nietzsche y Heidegger sobre la historia, Foucault adhiere a aquellas posiciones que 
juegan al dramático heroísmo del presente ${ }^{5}$. Pese a su intención de desmontar el sujeto soberano y exorcizar el mito del origen trascendental para salir de la antropología, Foucault está atrapado en una especie de metafísica de la historia que oscuramente deja a un punto de vista suprahistórico hacer su juego. En esa década, parpadeaba Foucault cuando la crítica en su fascinación veía la senda solemne de nuevos amaneceres que, dramática y teatralmente, conducían la salida de noches oscuras. Entonces las nociones de «transgresión», "pensamiento del afuera», «resistencia» entre otras, se oponían a «represión», «exclusión», «violencia», «ideología» «ilusión», etc.; Heidegger, Nietzsche, Bataille, Blanchot, Sade y aun los locos contra Kant, Spinoza, Descartes, Aristóteles; centelleaba el sofista contra el filósofo. "Sous les pavés, la plage". Es tal la fuerza de ese dramatismo que Foucault también invitará a otros crímenes filosóficos, como acaece en su curso Lecciones sobre la voluntad de saber: "para escapar a la trampa de Kant, hay que matar a Spinoza [...]. No se puede escapar de Kant más que después de estar liberado de Spinoza" (Foucault, 2011, p. 28). (Matar a Spinoza; o matar al hombre). A ese punto de vista, Foucault hace una autocrítica fuerte, en 1983:

[...] posiblemente haya que decir de los hábitos los más nocivos del pensamiento contemporáneo [...]: el análisis del momento presente como si éste fuera precisamente el momento de la ruptura en la historia, o el de la cima, o el del cumplimiento, o el de la aurora que vuelve. La solemnidad con que toda persona que mantiene un discurso filosófico reflexiona su propio momento me parece un estigma. Digo esto, sobre todo, porque yo mismo lo he hecho [...] porque en alguien como Nietzsche se encuentra, sin tregua o, al menos, de forma bastante insistente. Creo que hay que tener la modestia de decirse que, por una parte, el momento en el que uno vive no es ese momento único, fundamental, o irruptivo de la historia, a partir del cual todo se acaba y todo recomienza [...] (Foucault, 2001b, p. 1267).

La formulación de la muerte del hombre queda incluida en ese estigma, en esa solemnidad dramática con que se reflexiona el propio momento como si este fuera el momento de la ruptura en la historia y de la aurora que vuelve. En ese punto de vista suprahistórico, los dualismos hacen su mejor juego: un «lado bueno» se opone exteriormente a un «lado malo».

${ }^{5}$ Dreyfus y Rabinow quienes -en la primera parte de su libro Michel Foucault Un parcours philosophique-, al estudiar su pensamiento de la década de los años sesenta, remiten constantemente sus problemas y ensayos a Heidegger: "en este estadio, y sin decirlo, Foucault se ha sumergido en los abismos más profundos del comienzo del pensamiento heideggeriano"(Dreyfus y Rabinow, 1984, p. 63). 
La salida del dualismo variopinto se efectúa progresivamente Foucault va descubriendo en sus nuevos estudios la insostenibilidad de ciertas nociones usadas como categorías en análisis pasados. Seguidamente al curso Lecciones sobre la voluntad de saber, las nociones de exclusión, represión, transgresión, violencia, institución, se van borrando progresivamente. Por ejemplo, en el curso La sociedad punitiva (1972-1973), un objeto lateral y permanente es la crítica a una noción que Foucault ha usado con frecuencia: "esta noción de exclusión me parece, de entrada, muy abierta y, sobre todo recompuesta y artificial. Lo digo en cuanto que yo mismo la he usado y, posiblemente, abusado" (Foucault, 2013b, p. 4). Y unas páginas después agrega: "posiblemente sería necesario, del mismo modo, hacer la crítica de una noción cuya fortuna le ha sido correlativa: aquella de transgresión [...]. Me parece que las direcciones que indican los análisis adelantados en términos de exclusión y de transgresión deben ser continuadas bajo dimensiones nuevas [...]" (id., 7). Notorio desplazamiento respecto al curso Lecciones sobre la voluntad de saber, donde uno de sus puntos mayores es la exclusión del sofista y la voluntad tácita de hacer valer su transgresión.

La composición de la noción poder-saber, y los análisis que conduce, pone a la vista la imposibilidad de cualquier tipo de exterioridad. El pensamiento del «afuera», con sus derivaciones (represión, exclusión, transgresión, etc.) y sus operaciones, se hace insostenible: "es ilusión creer que la locura -o la delincuencia, o el crimen- nos habla a partir de una exterioridad $[\ldots]$ se está siempre en el interior. El margen es un mito. La palabra de afuera es un sueño que uno no cesa de reconducir" (Foucault, 2001c, p. 77). En síntesis, el despliegue progresivo de la noción poder-saber en los análisis de diferentes dominios muestra a Foucault que los dos lados son una ilusión del pensamiento. Si en ciertas coyunturas los dualismos simplificadores han sido útiles, luego ha de venir el momento de un nuevo desplazamiento que los rompe. En 1977, Foucault dice: "hay que pasar al otro lado -del lado bueno-, pero para ensayar desprenderse de esos mecanismos que hacen aparecer dos lados, para disolver la falsa unidad, la naturaleza ilusoria de ese otro lado por el cual uno ha tomado partido. Es ahí donde comienza el verdadero trabajo, aquel del historiador del presente" (Foucault, 2001d, p. 265). Sin duda, la formulación de la muerte del hombre en Las palabras y las cosas se inscribe bajo el signo de esos mecanismos que hacen aparecer dos lados. Cuando el pensamiento devela la naturaleza ilusoria y el falso valor del «lado bueno», comienza el trabajo efectivo de la historia crítica del presente: 
Me pregunto si no se podría caracterizar uno de los grandes roles del pensamiento filosófico justamente a partir de la pregunta kantiana «Was ist Aufklärung?», diciendo que la tarea de la filosofía es decir qué es el hoy y decir qué «somos nosotros hoy». Pero no concediéndonos la facilidad algo dramática y teatral de afirmar que este momento en el que nos encontramos es, en lo más profundo de la noche, el de la perdición más grande, o en el despuntar del día, aquel en el que el sol triunfa, etc. (Foucault, 2001b, p. 1267).

Cuando empieza el verdadero trabajo del historiador del presente, se deshace el dualismo del dramatismo de la negra noche y la solemnidad de la nueva aurora. La muerte del hombre, tal como se planteara otrora, resulta insostenible en un historia efectiva del presente. Tal vez de ella solamente subsisten dos cosas: la metáfora, que designa el querer poner fin a aquellos discursos de verdad que apuntan a la producción del hombre por el hombre, y la historicidad de la producción del sujeto y la subjetividad. Así, el pensamiento al volver su vista atrás ve que el hombre es solamente un episodio de la historia de la verdad, un acontecimiento de la cultura occidental, en la historia de las relaciones subjetividad y verdad, en la historia incesante y $\sin$ fin de sus transformaciones, innovaciones y recomposiciones.

\section{La modestia histórica del presente}

En sus últimos años de trabajo, Foucault opera un nuevo desplazamiento de su pensamiento: problematización histórico-crítica de las técnicas; y ahí, la cuestión del hombre no está exenta de los efectos inducidos. A propósito de su trabajo, dice, en 1980: "en suma, el objetivo de mi proyecto es construir una genealogía del sujeto moderno. El método es un arqueología del saber, y el dominio preciso del análisis es lo que yo debo llamar tecnologías: quiero decir la articulación de ciertas técnicas y de ciertos tipos de discursos a propósito del sujeto" (Foucault, 2013a, p. 36-37). Se conoce que el gran desafío del pensamiento de Foucault fue salir de la filosofía del sujeto trascendental, cuya salida la opera a través de la elaboración de una genealogía del sujeto moderno, estudiando su constitución (y no deconstruyéndolo) a través de la historia, de una historia que nos ha conducido al concepto moderno de sí (en el que está implicada la antropología). Puesto que, en la historia de la cultura occidental, las prácticas por las cuales el sujeto es definido y transformado se han acompañado de ciertos tipos de saber, de discursos de verdad, la genealogía del sujeto es concomitante con una historia del poder de la verdad, del gobierno de los hombres a través de la verdad. En el marco de su proyecto, Foucault, 
inicialmente se interesó por la prácticas discursivas modernas, que producen una serie de discursos de verdad sobre el sujeto en general; seguidamente, por las prácticas no discursivas que producen al mismo tiempo el sujeto como objeto de saber y objeto de dominación; y, por último, por las prácticas de sí a través de las cuales el sujeto produce un conocimiento de sí mismo. En los últimos años de la década del 70, y a partir de Habermas, Foucault modifica su punto de vista sobre varios aspectos importantes. Retoma las técnicas como una cuestión mayor si se desea estudiar el sujeto. En la cultura occidental se puede distinguir tres tipos de técnicas: técnicas de producción de objetos; técnicas de significación a través de las cuales se produce el lenguaje, el saber, el discurso; y técnicas de dominación, a través de la cuales se gobierna a los individuos sometiéndolos a ciertos fines, objetivando el sujeto. Estudiando las prácticas a través de las cuales los individuos producen un conocimiento sobre ellos mismos, Foucault descubre otro tipo técnica: técnicas de sí. Estas sostienen una relación constante con las técnicas de dominación, las dos se entre-tienen, su encuentro constituye la noción de gobierno $^{6}$. La apuesta de una filosofía histórico-crítica gana claramente su impronta política: una política de nosotros mismos. Foucault modifica su punto de vista, el proyecto de la genealogía del sujeto moderno se somete a ciertos ajustes: introduce retrospectivamente la noción de técnicas en los segmentos desarrollados de su proyecto y también en lo que está efectuando (y hasta su muerte). La noción de técnica deviene en un aspecto importante de su pensamiento. ¿Por qué razón? Lo real no es racional ni lo racional es real; lo real no es otra cosa que lo que hacen los hombres, las prácticas. Las prácticas (lo real e irracional) producen técnicas coherentes, reflexivas, con objetivos definidos y amarradas a la obligación de producir sujetos y objetos, a buscar y decir la verdad. Las técnicas van a marcar con la impronta de la verdad lo que hacen los hombres.

Retomemos, el proyecto de Foucault es la construcción de una genealogía del sujeto moderno, en ella emplea la arqueología del saber y fija como dominio específico del análisis las tecnologías, en las cuales incluye las técnicas de sí. Se infiere entonces que la cuestión del hombre como dominio preciso de análisis se desplaza al dominio de las tecnologías. Por otra parte, si en la década de los años 60, la cuestión del hombre y su muerte, se conducen bajo la luz de los trabajos de Heidegger (y a la par

6"Yo creo que si uno va a estudiar la genealogía del sujeto en la civilización occidental, hay que tener en cuenta no solamente las técnicas de dominación, sino también las técnicas de sí [...]. El punto de contacto donde [el modo por el cual] los individuos son dirigidos por otros se articula con la manera con la cual ellos se conducen a sí mismos, es lo que, creo, puedo llamar «gobierno»" (Foucault, 2013a, pp. 38-39). 
con Nietzsche, como se consideró anteriormente), veinte años después, Foucault opera una inversión eminente sobre lo que antes había tomado del filósofo alemán:

Para Heidegger, era a causa de una obsesión creciente por la techné que, como el solo medio de llegar al conocimiento de los objetos, Occidente ha perdido el contacto con el Ser. Retornemos la cuestión y preguntémonos cuáles técnicas y cuáles prácticas forman el concepto occidental del sujeto, dándole su cesura característica de la verdad y del error, de la libertad y de la constricción. Creo que es allá que encontramos la real posibilidad de construir una historia de lo que hacemos y, al mismo tiempo, un diagnóstico de lo que nosotros somos [...]. En suma, se trata de buscar otro tipo de filosofía crítica [...] una filosofía crítica que busca las condiciones y las posibilidades indefinidas de una transformación del sujeto, de nuestra propia transformación (Foucault, 2013a, pp. 36- 37).

La operación es grave. A partir de ahí, Foucault reconstruye el diagnostico de lo que nosotros somos en la actualidad, una ontología histórica de nosotros mismos, es decir: una filosofía crítica que busca las condiciones y posibilidades de la transformación de lo que somos, del sujeto, del hombre. Mas se trata de condiciones y posibilidades «indefinidas», y no de la próxima muerte del sujeto. Foucault puede reinscribir todo el proyecto de sus investigaciones bajo la dimensión de las prácticas y las técnicas (lo que no implica que las técnicas se reduzcan a las técnicas de sí, puesto que estas constituyen solamente uno de sus tipos). La puesta en cuestión de la antropología queda inscrita en el dominio específico de las técnicas. Desde fines de la década del 70, Foucault enfoca su trabajo sobre las prácticas y técnicas que conducen al ser humano a construir, o transformar, o, reforzar, su subjetividad, a constituirse en sujeto de sí mismo, en sujeto moral. Mas tal focalización no consiste en el pasaje de una operación de deconstrucción del sujeto a la reconstrucción del sujeto por sí mismo; se trata más bien de la introducción del tercer haz de relaciones que, junto a los saberes y el poder, conforman el pensamiento y le permiten pensar su propia historia. Foucault jamás somete a ningún desplazamiento los haces de relaciones del pensamiento ${ }^{7}$.

${ }^{7}$ En el curso El gobierno de los vivos, se plantea la necesidad de un desplazamiento, de desplazarse de la noción saber-poder hacia la noción verdad-gobierno, pero ahí se trata de un desplazamiento en el interior del mismo haz, y no de un haz a otro diferente (del poder a la cuestión del gobierno y la del saber a la cuestión de la verdad, en función de alcanzar mayor precisión) (cf. Foucault, 2012, p. 13). 
Ahora bien, si nos situamos en el proyecto de una genealogía del sujeto moderno -en el que el método es una arqueología del saber y el dominio preciso del análisis, las tecnologías-, enfocándonos en la faz de la constitución del sujeto por el individuo mismo, en el dominio de análisis de las técnicas de sí, ¿qué acaece con la antropología?, ¿se borran como un rostro de arena en la orilla del mar? No, ahí, estas cuestiones se abordan bajo el foco de lo que se puede llamar la otra faz de las problematizaciones (y poniendo en jugo las rectificaciones hechas a la muerte del hombre). Foucault considera que las ciencias humanas suponen como relación principal de la subjetividad (la relación que un individuo instaura consigo mismo) una relación de conocimiento, de interpretación de un sí escondido: "pero el sí, creo, no debe ser considerado como una realidad que puede estar escondida; creo que el sí debe ser considerado como el correlato de tecnologías desarrolladas a través de nuestra historia" (Foucault, 2015, p. 98). Entonces, hoy, el problema ya no es proclamar la próxima muerte del hombre, sino la necesidad de reinventar otras técnicas diferentes de las creadas por el cristianismo de los primeros siglos, diferentes a la hermenéutica, de las que las ciencias humanas y sus antropologías son las herederas. Respecto a este punto, es pertinente considerar, de manera sucinta y esquemáticamente, cómo la invención del hombre, de la antropología y hasta cierto punto de las ciencias humanas, procede de un tipo de técnica inventada por el cristianismo.

El hombre emerge de una matriz cristiana. En la cultura occidental, Foucault descubre dos grandes matrices que ordenan los fines que conducen los modos de subjetivación y las técnicas de sí: primera, cuando el principio «preocúpate de ti mismo» toma la forma de un dominio de sí sobre sí mismo; segunda, cuando ese principio pasa a tomar la forma de un conocimiento de sí por sí mismo. Las técnicas de sí del mundo greco-romano apuntan a producir un sujeto moral positivo, mientras que técnicas de sí del cristianismo apuntan a producir un tipo de sujeto marcado por el conocimiento de sí sobre sí mismo y en función de su propio sacrificio. En esta última matriz se sitúa el origen histórico de las tentaciones antropológicas de la cultura occidental y de nuestra subjetividad contemporánea. En la subjetividad grecorromana, la verdad no se descubre en las características personales del sujeto, ella está en función de constituir un tipo de sí que puede tomar la forma de sujeto de conocimiento o sujeto de voluntad. En esa experiencia, una interpretación, un examen profundo y exhaustivo, mediante el cual el sujeto diga la verdad oculta de sí, y de tal manera que el maestro pueda ejercer sobre él un gobierno de su conducta, no es indispensable ni importante, como en el cristianismo. El cristianismo retoma un buen número de prácticas de sí greco-romanas (el examen de sí y la confesión cristiana 
tienen antecedentes en el mundo antiguo), mas borra las condiciones de posibilidad de la producción positiva de la subjetividad. En la antigüedad, el foco de las inquietudes es la relación con las cosas, mientras que en el cristianismo son los secretos de la conciencia, que hay que descifrar. Un nuevo tipo de sujeto moral se ha producido. El sí ya no es algo a construir, sino algo a descifrar para sacrificarlo.

En el cristianismo de los primeros siglos, Foucault ve que todos y cada uno de los individuos tienen el deber de saber lo que cada uno es, a conocer lo que pasa en sí mismo, conocer las tentaciones a las cuales se ha expuesto y está obligado a decir esas cosas a otros, a dar testimonio contra sí mismo a partir de ellas. Uno de los modos de producir la verdad de sí es la confesión, exagoreusis. En la confesión, el examen de sí se opera, en primer lugar, cuando el monje confiesa continuamente el movimiento de sus pensamientos, mas el momento hermenéutico decisivo es cuando el monje mismo, confesando la verdad, hace aparecer el signo de la verdad del origen real de sus pensamientos. La exagoreusis: una hermenéutica de sí. La verbalización constituye el medio privilegiado para seleccionar los pensamientos, para separar su autenticidad o su falsedad. Verbalizar el movimiento de los pensamientos ante otro es el medio de poner todas las ideas bajo los ojos de Dios, y así descubrir lo que ellos son. La verbalización: un movimiento hacia Dios, un movimiento de renuncia a sí, de sacrificio de sí (cf. Foucault, 2013a, p. 86). Las técnicas orientadas hacia el descubrimiento y la formulación de la verdad de sí, hermenéuticas, despiertan el interés de Foucault ${ }^{8}$. Estas técnicas juegan un papel político importante, después de la Edad Media:

Quisiera mostrar la transformación [...] del viejo precepto délfico «conócete a ti tú mismo» (gnôthi seauton) en el precepto monástico «dime cada uno de tus pensamientos» (omnes cogitationes). Pues este precepto, nacido y desenvuelto primero las instituciones monásticas, ha jugado, creo, un rol importante en la constitución de la subjetividad moderna (Foucault, 2013b, pp. 40-41).

El cristianismo instaura dos gruesos tipos de tecnologías de la verdad sobre sí que oscilan entre una tendencia ontológica y una tendencia epistemológica: la primera apunta a la manifestación del ser del pecador, y la otra, a un análisis discursivo y permanente del pensamiento, exagoreusis; en

8 “[...] Foucault ha concentrado sobre todo su atención sobre la exagoreusis, precisamente porque ella ha predominado sobre las otras y es todavía la forma dominante de nuestra «hermenéutica de sí»" (Davidson, 2013, p. 68). 
ambas está en juego el sacrificio de sí. La tecnología de orden hermenéutico, epistemológico, tras los avatares de siglos de conflictos y fluctuaciones, ha llegado a ser dominante en la actualidad, se ha constituido en una experiencia histórica en la cultura occidental, con su propia problematicidad.

Creo que uno de los grandes problemas de la cultura occidental ha sido encontrar la posibilidad de fundar la hermenéutica de sí no como era en el caso del cristianismo primitivo, sobre el sacrificio de sí, sino al contrario, sobre una emergencia positiva, sobre la emergencia teórica y práctica del sí. Esta era la meta de las instituciones judiciales, esta era también la meta de las prácticas médicas y psiquiátricas, esta era la meta de la teoría política y filosófica -constituir el fundamento de la subjetividad en tanto que raíz de un sí positivo, lo que podríamos llamar el antropologismo permanente del pensamiento occidental-. Y creo que este antropologismo está atado con el profundo deseo de substituir el sacrificio de sí que, para el cristianismo era la condición de apertura del sí en tanto que campo de interpretación indefinida, por la figura positiva del hombre. En el transcurso de los dos últimos siglos, el problema ha sido: ¿cuál podría ser el fundamento positivo de las tecnologías de sí que nosotros hemos desarrollado durante siglos? Pero, posiblemente, llega el momento para nosotros de preguntarnos si tenemos verdaderamente necesidad de esta hermenéutica de sí. Puede ser que el problema de sí no sea descubrir un sí positivo o el fundamento positivo de sí. Puede ser que, ahora, nuestro problema sea descubrir que el sí no es otra cosa que el correlato histórico de la tecnología construida en el curso de nuestra historia. Puede ser que el problema sea cambiar esas tecnologías. Y en este caso, uno de los principales problemas políticos, hoy día, sería, en sentido estricto de la palabra, la política de nosotros mismos (Foucault, 2013b, pp. 90-91).

Este fragmento permite destacar varios puntos-clave. 1) Tras siglos de avatares, la tecnología de sí de orden hermenéutico ha llegado a ser dominante en Occidente. Mas, en el cristianismo, tenía por finalidad el sacrificio de sí: "paradoja ética del ascetismo cristiano". 2) En los tiempos modernos, esa paradoja da lugar a un problema grande: la experiencia histórica moderna de la subjetividad, por una parte, apropia, sostiene y actualiza la hermenéutica de sí cristiana; pero, por otra, se esfuerza por deshacer su paradoja, se aplica a sustituir la renuncia de sí por un sí positivo: antropología. Una ruptura decisiva, un nuevo tipo de sujeto se produce, «el hombre» ${ }^{9}$. 3) Situado en la experiencia histórico-crítica de las tecnologías de sí, Foucault descalifica

9 “A partir del siglo XVIII y hasta la época presente, las «ciencias humanas» han reinsertado las técnicas de verbalización en un contexto diferente, haciendo de ellas no el instrumento de la renuncia del sujeto a él mismo, sino el instrumento positivo de la 
la necesidad esa hermenéutica de sí. El problema mayor entonces no es descubrir o fundar la positividad del «hombre», sino descubrir que el sí, o el «hombre», nuestra subjetividad contemporánea, solo es el correlato histórico de un tipo de tecnología que se ha venido constituyendo desde el cristianismo de los primeros siglos. Por consiguiente, el problema de hoy sería crear otros tipos de tecnologías que no sean de orden hermenéutico. El blanco de la crítica se desplaza del hombre, o la antropología, al tipo de tecnología que lo produce. Así, uno de los principales problemas políticos de hoy es la política de nosotros mismos (inferencia que resulta clara si se tiene en cuenta aquello que designa el concepto de gobierno).

\section{Conclusiones}

Parece plausible considerar que el proyecto de Foucault es la genealogía del sujeto moderno. Si se tiene en cuenta que la constitución del sujeto moderno comporta un carácter antropológico, se infiere que "el hombre" está implicado en el proyecto de esa historia. Pero, es necesario reconocer que el modo como Foucault asume la historia, y el tiempo presente en ella, cambia significativamente a través de las experiencias de su pensamiento. Doce años después de la publicación de Las Palabras y Las cosas, el filósofo reconoce que se equivocó al presentar la "muerte del hombre" como algo que acontecía en la actualidad; sin embargo, manifiesta no ceder en el fondo de la cuestión, es decir, en lo que se refiere a su rechazo a la antropología y a la invención de la figura del hombre. En la década del 60 -conducido por una lectura de Nietzsche-, Foucault definía la filosofía como un diagnóstico del presente, que rompe dramáticamente con el pasado. El presente, a partir del acontecimiento histórico que lo instaura, emerge como una nueva aurora, por ejemplo: "Y he aquí que en este Pliegue, la filosofía se ha adormecido con sueño nuevo; no ya el del Dogmatismo, sino el de la Antropología [...]. Para despertar al pensamiento de un sueño tal -tan profundo que lo experimenta paradójicamente como vigilia [...]-, para llamarlo a sus posibilidades más matinales, no hay otro medio que [...]" (Foucault, 1966, pp. 352-353). Bajo esa perspectiva, Foucault anunciaba solemnemente el fin del "hombre" como el acontecimiento que definía la actualidad. Mas, en el correr de los últimos años de la década del 70 -conducido por una lectura de Kant-, cambia su modo de asumir la historia y la actualidad: adviene la modestia del presente. El presente es como todos los otros presentes que han transcurrido en la historia; el presente no es ni una noche que termina ni una nueva aurora del pensamiento. En esta nueva perspectiva, el filósofo constitución de un nuevo sujeto. Que la utilización de esas técnicas haya cesado de implicar la renuncia del sujeto a él mismo constituye una ruptura decisiva" (Foucault, 2001e, p. 1632). 
no admite una supuesta nueva aurora del pensamiento que se abriría con la muerte del hombre; mientras que la antropología y el hombre se reinscriben como un episodio contemporáneo en la larga historia de las técnicas de sí inventadas por el cristianismo de los primeros siglos.

\section{Referencias bibliográficas}

Castro, R. (2005). La frase de Foucault: el hombre ha muerto. Alpha (Osorno), (21), 225-233. doi: 10.4067/S0718-22012005000100015.

Castro, E. (2006). Michel Foucault: sujeto e historia. Tópicos, (14), 171-183.

Davidson, A. (2013). La fin de l'herméneutique de soi. En D. Lorenzini, A. Revel, y A. Sforzini (dirs.), Michel Foucault: éthique et vérité 1980-1984 (pp. 67-76). Paris, Francia: Vrin.

Dreyfus, H., y Rabinow, P. (1984). Michel Foucault Un parcours philosophique, Au-delá de l'objectivité et de la subjectivité (F. Durand-Bogaert, Trad.). Paris, Francia: Gallimard.

Foucault, M. (1966). Les mots et les choses. Une archéologie des sciences humaines. Paris, Francia: Gallimard.

Foucault, M. (2001a). Entretien avec Michel Foucault (avec D. Trombadori, 1978).

En D. Defert, F. Ewald, y J Lagrange (Eds.), Dits et écrits II, 1976-1988 (pp. 860-914). Paris, Francia: Gallimard.

Foucault, M. (2001b). Structuralisme et poststructuralisme (entretien avec Raulet, 1983). En D. Defert, F. Ewald, y J Lagrange (Eds.), Dits et écrits II, 1976-1988 (pp. 1250-1276). Paris, Francia: Gallimard.

Foucault, M. (2001c). L'extension sociale de la norme" (entretien avec P. Wermer, 1976). En D. Defert, F. Ewald, y J. Lagrange (Eds.), Dits et écrits II, 1976-1988 (pp. 74-79). Paris, Francia: Gallimard.

Foucault, M. (2001d). Non au sexe roi (entretien avec B.- H. Lévy, 1977). En D. Defert, F. Ewald, y J. Lagrange (Eds.), Dits et écrits II, 1976-1988 (pp. 256269). Paris, Francia: Gallimard.

Foucault, M. (2001e). Les techniques de soi. En D. Defert, F. Ewald, y J. Lagrange (Eds.), Dits et écrits II, 1976-1988 (pp. 1602-1632). Paris, Francia: Gallimard.

Foucault, M. (2011). Leçons sur la volonté de savoir. Cours au Collège de France. 1970-1971 suivi de Le savoir d'Edipe. Paris, Francia: Gallimard - Seuil.

Foucault, M. (2012). Du gouvernement des vivants. Cours au Collège de France. 1979-1980. Paris, Francia: EHESS - Gallimard - Seuil.

Foucault, M. (2013a). L'origine de l'herméneutique de soi. Conférences prononcées à Dartmouth College, 1980 (H. P. Fruchaud y D. Lorenzini, Eds). Paris, Francia: Vrin.

Foucault, M. (2013b). La société punitive. Cours au Collège de France. 1972-1973. Paris, Francia: EHESS - Gallimard - Seuil.

Foucault, M. (2015). Qu'est-ce que la critique? Suivi de La culture de soi. Paris, Francia: Vrin.

Gros, F. (2007). Michel Foucault. Paris, Francia: Puf. 
Margot, J. P. (2015). Michel Foucault : La revolución y la cuestión del presente. Praxis Filosófica, (41), 193-214. doi: 10.25100/pfilosofica.v0i41.3187.

Nigro, R. (2012). Le grondement de la critique du sujet fondateur dans le réveil du sommeil anthropologique. Rue Descartes, 75(3), 60-71. doi: 10.3917/ rdes.075.0060. 
